\title{
Self-activated photoblinking of nitrogen vacancy centers in nanodiamonds (sandSTORM): A method for rapid single molecule localization microscopy with unlimited observation time
}

\author{
Kaarjel K. Narayanasamy ${ }^{a}$. Joshua C. Price ${ }^{b}$, Raquel Mesquita-Riberio ${ }^{c}$, Melissa L. Mather ${ }^{\text {,** }}$ \\ and Izzy Jayasinghe ${ }^{\text {a,d,* }}$
}

\begin{abstract}
${ }^{a}$ Faculty of Biological Sciences, University of Leeds, Leeds LS2 9JT, United Kingdom
${ }^{\mathrm{b}}$ Optics and Photonics Research Group, Faculty of Engineering, University of Nottingham, Nottingham, NG7 2RD, United Kingdom

${ }^{c}$ School of Life Sciences, Medical School Building, University of Nottingham, Nottingham, NG7 2UH, United Kingdom

${ }^{\mathrm{d}}$ Department of Molecular Biology and Biotechnology, Faculty of Science, The University of Sheffield, Sheffield, S10 2 TG, United Kingdom
\end{abstract}

* Correspondence: Dr I. Jayasinghe (‥jayasinghe@sheffield.ac.uk) and Prof. M. L. Mather (melissa.mather@nottingham.ac.uk)

\begin{abstract}
Stochastic optical reconstruction microscopy (STORM) is one of the most commonly used super-resolution microscopy techniques. Popular implementations of STORM utilize aromatic fluorophores and consist of a number of intrinsic limitations such the finite photostability of the dyes, the reliance upon non-physiological redox buffers and speed which is ultimately limited by the 'off'rates of the photoblinking. Self-activated nanodiamond-based STORM (sandSTORM) has been developed as an accelerated STORM protocol which harvests the rapid, high quantum-yield and sustained photoblinking of nanodiamonds (ND). Photoluminescence emanating from the stochastic charge-state interconversion of Nitrogen Vacancy (NV) centers between NV ${ }^{0}$ and $\mathrm{NV}^{-}$is localized using conventional STORM-optimized hardware and image processing protocols over an unlimited duration of imaging. This produces super-resolution images of matching resolution at $\sim 3$-times the speed and $\sim 100$ times less light exposure to the sample compared to traditional STORM. The enabling NDs have been used to map arrays of ryanodine receptor in skeletal muscle tissues via immunolabelling and directly visualize the internal spaces of living neurons via endocytosis of NDs. This paper details the physical basis of sandSTORM, factors which optimize its performance, and key characteristics which make it a powerful STORM protocol suitable for imaging nanoscale sub-cellular structures.
\end{abstract}

Keywords: single molecule localization microscopy (SMLM), super-resolution microscopy, stochastic optical reconstruction microscopy (STORM), nanodiamonds, nitrogen vacancy

\section{Introduction}

Super-resolution microscopy has transformed cell and molecular biology by breaking the diffraction limit of light enabling biological structures critical to cell function and life to be resolved at a molecular scale. Commonly-visualized structures include cellular organelles [1, 2], cytoskeleton $[3,4]$, nucleic acid organization $[5,6]$ and protein clustering $[7,8]$; see review [9]. Among many super-resolution techniques which continue to emerge and evolve, single molecule localization microscopy (SMLM) is arguably the most popular modality used by life scientists, with the variant known as STORM widely used, largely owing to its compatibility with well-established fluorescent marker probes and immuno-labelling protocols [10-12]. The basis of STORM is the separation of consecutively emitting fluorescent sources within diffraction-limited spots from sequential images. Post processing is performed to localize fluorescent reporters within a single reconstructed image using centroid-localization algorithms and Gaussian fitting methods achieving precision down to $\leq 20 \mathrm{~nm}$ [13]. Thousands of images are typically acquired to improve localization precision based on activation of stochastically different fluorescent emitters within diffraction limited spots [14].

A fundamentally important element of attaining high quality super resolution images via STORM is the use of fluorescent probes that exhibit at least two states that last for sufficient time to enable these to be distinguished as 'on' and 'off' [14]. Fluorescent probes typically used in STORM include organic fluorophores, fluorescent proteins and solid- 
Narayanasamy et al., 18 MAY 2020 - preprint copy - BioRxiv

state nanoparticles. The photophysical behavior of such probes can be controlled via multiple mechanisms including light induced interconversion between different spectroscopic states [11, 15], chemically mediated blinking $[16,17]$, reversible photoisomerization of chemical groups [18] and transient binding and unbinding of probes [19, 20]. Fluorescent probe selection and design requires consideration of probe brightness and the ratio between the brightness of the probe in the 'on' and 'off' states, as these parameters have a significant impact on the localization precision [14]. The photostability, number of cycles over which the probe can change state and the switching duty cycle, are also important factors affecting image quality, acquisition time and the available measurement window.

Efforts to improve fluorescent probes for localization microscopy are ongoing. Recent refinements tackle issues such as the availability of switchable probes, integration of protocols with established labelling methods, the rapid photobleaching of organic probes and the intrinsically poor temporal resolution of such imaging experiments which owes to the need to acquire thousands of images. Many of these shortcomings have been addressed using direct STORM (dSTORM) which is a method that enables the use of conventional fluorescent probes such as labelled antibodies or chemical dyes (e.g. cyanine dyes) without the need for additional activator fluorophores, required in previous methodologies [17].

Research is also underway to address the limited quantitative capacity of STORM arising as a result of the lack of control over fluorophore stochastic photo-switching and photo-bleaching [16, 21, 22] in addition to the inherent slowness of the imaging modality. Further advances in STORM have been made through the implementation of alternative probes based on solid-state nanoparticles such as pure metal nanoparticles [23], lanthanide-doped nanoparticles [24], carbon dots [25] and quantum dots [26]. Advantageously these probes have been demonstrated to address the rapid photobleaching which occurs in organic fluorophores opening the door to time course and repeated studies.

Currently, STORM is more commonly implemented using fixed cells however there is tremendous interest in establishing STORM techniques that enable live cell time course imaging. This demands fast cycling fluorescent probes that are resistant to photobleaching, produce high contrast between the 'on' and 'off' states, and in the case of intracellular imaging can be transported across the cell membrane. The realization of this will reduce the number of images required for reconstruction and increase the achievable frame rate, minimizing motional artefacts and increasing sample throughput. Typical STORM imaging times for fixed samples are of the order of 20-30 minutes and to meet the demands of live cell imaging and/or high-throughput imaging, these times need to be reduced by at least an order of magnitude. Allied to work centered on fluorescent probe optimization, are studies seeking to increase imaging speeds based on algorithmic enhancement of the image reconstruction and through the establishment of algorithms to accommodate either high [27, 28] or sparse marker localization densities [29]. Conspicuously lacking however, have been experimental approaches which offer genuine acceleration of STORM through a reduction of the time between localizations (i.e. 'off' times) without compromising the resolution or the durability of the samples.

Herein a method for rapid SMLM with unlimited observation time based on self-activated photoblinking of Nitrogen Vacancy (NV) centers in nanosized diamond (nanodiamonds (ND)) is reported. The NV center is a naturally occurring paramagnetic impurity comprising a substitutional Nitrogen atom adjacent to a vacant site in the diamond lattice. This defect has been reported to exist in three different charge states namely the negative charge state $\left(\mathrm{NV}^{-}\right)$, neutral charge state $\left(\mathrm{NV}^{\circ}\right)$ and positive charge state $\left(\mathrm{NV}^{+}\right)$, defined by the number of unpaired electrons nearby the defect. The $\mathrm{NV}^{-}$center in particular has attracted attention as a potential fluorescent probe for use in biological imaging due to its high quantum yield, robust luminescence, which does not bleach, and the inherent low cytotoxicity of diamond $[30,31]$. The method presented here leverages the intrinsically-driven interconversion between the $\mathrm{NV}^{\circ}$ and $\mathrm{NV}^{-}$charges states mediated via photoionization and the surrounding chemical environment [31]. Photoluminescence of NV centers has been previously been used for deterministic super-resolution techniques such as Stimulated Emission Depletion (STED) and Ground State Depletion (GSD) microscopies $[15,32,33]$. To use them as probes for localization microscopy, it has always been necessary to manipulate the spin transitions of NV centres with the use of extrinsic microwave which has limited their utility in imaging biological nanostructures [30, 34-36]. The present work is the first time, to the authors' knowledge, SMLM based on the stochastic blinking of NVs has been demonstrated on biological samples. In particular, the intracellular ryanodine receptor (RyR) ion channel is imaged within skeletal muscle tissue using antibody conjugated NDs containing NVs. Additionally, endocytosed nanodiamonds are localized with super-resolution in networks of neuronal cells. Further, this work demonstrates how NVs can be photoswitched at very short dark times by carefully controlling the emission wavelength used for localization and the electrolyte solution samples are immersed in. Significantly, sandSTORM is shown to enable up to 10 times higher frames rates (100 fps compared with 10-20 fps) than dSTORM with the added advantages of simpler probe preparation, compatibility with basic cell culture buffers and use of probes that are resistant to photobleaching.

\section{Results and Discussion}

\section{sandSTORM}

\section{Super-resolution localization of ion channel arrays}

Time-sequential wide field images of transverse cryosections of extensor digitorum longus (EDL) muscles from rats were acquired based on the photoluminescence from NVs within commercially sourced NDs colocalized with RyR calcium release channels. Experimentally NVs were excited using a $561 \mathrm{~nm}$ laser line and the resulting photoluminescence within a $605 \mathrm{~nm} \pm 35 \mathrm{~nm}$ pass band used to form individual image frames. Post processing of $\sim 30,000$ images was subsequently performed to reconstruct the RyR array networks with super-resolution based on the use of self-activated photoblinking of NVs within proximal NDs, the results of which are shown in Figure 1. The architecture typical of RyRs in transversely sectioned skeletal muscle fibers is faithfully depicted through a comparison with a corresponding diffraction limited wide-field fluorescent image (Figure 1 inset). The enhanced localization sandSTORM produces is apparent and the nanoscale morphology that it reports of the RyR arrays reproduces the observations of previous investigations using standard dSTORM [37].

The ability to resolve RyR arrays based on photoblinking from NVs using different spectral ranges of emitted light was also investigated based on the hypothesis that photoblinking is linked to NV charge state conversion between the $\mathrm{NV}^{\circ}$ and $\mathrm{NV}^{-}$states. These charge states have intrinsically different electronic structures and correspondingly different emission spectra [38], which are characterized by narrow zero phonon lines at 575 $\mathrm{nm}$ and $637 \mathrm{~nm}$ for the $\mathrm{NV}^{\circ}$ and $\mathrm{NV}^{-}$charge states respectively and broad phonon lines extending as far as $750 \mathrm{~nm}$ for $\mathrm{NV}^{\circ}$ and $800 \mathrm{~nm}$ for $\mathrm{NV}^{-}$. Experimentally the contribution from each charge state on the reconstructed sandSTORM image was investigated through the use of five different emission filters ( $520 \mathrm{~nm} \pm 20 \mathrm{~nm}$ bandpass (BP), $575 \mathrm{~nm} \pm$ 


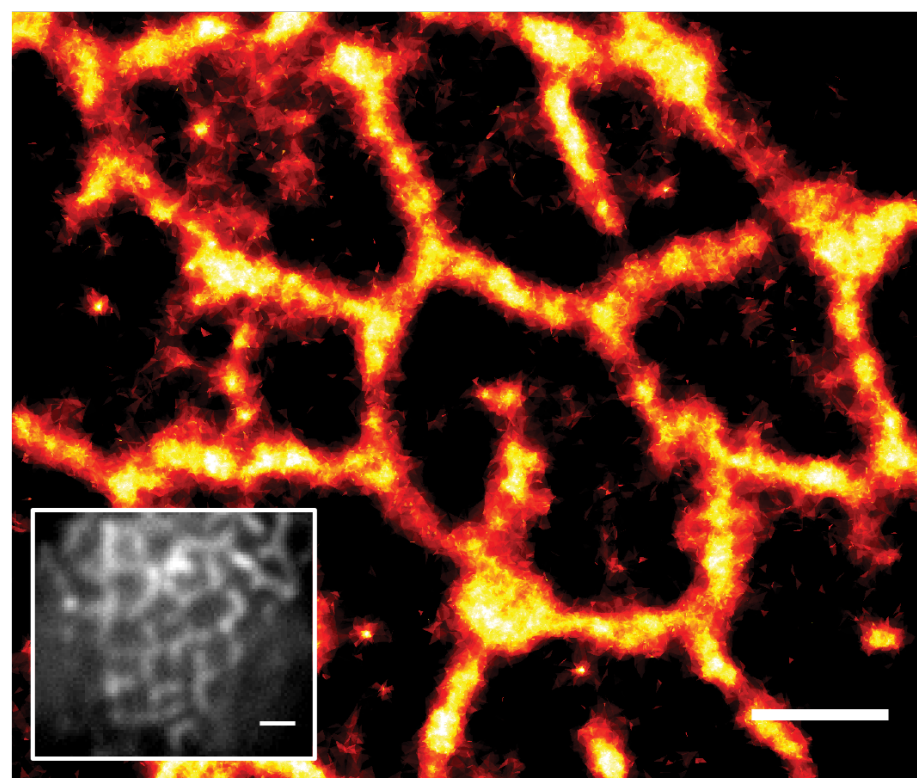

Fig. 1. sandSTORM image of RyRs labelled with ND-conjugated antibodies in a rat skeletal muscle transverse tissue section (scale bar $=1 \mu \mathrm{m})$. Inset displays diffraction limited image of an equivalent region (scale bar $=2 \mu \mathrm{m}$ )

$2.5 \mathrm{~nm}$ BP, $590 \mathrm{~nm}$ longpass (LP), $605 \mathrm{~nm} \pm 35 \mathrm{~nm} \mathrm{BP}, 692 \mathrm{~nm} \pm 20 \mathrm{~nm}$ BP) covering spectral ranges that selectively include or exclude contributions from the individual charge states. Figure 2 displays exemplar unprocessed images indicative of the photoluminescence detected using each emission filter (top row) and the resulting reconstructed sandSTORM images (bottom row).

Photoblinking was observed to be most prominent in the spectral band in which $\mathrm{NV}^{\circ}$ and $\mathrm{NV}^{-}$emission overlaps $(570 \mathrm{~nm}$ to $640 \mathrm{~nm}$ ). There was either insufficient blinking and/or inadequate contrast between the 'on' and 'off' states outside of these bands for sandSTORM images to be formed. Spectral windows extending well beyond these bands (e.g. 590 LP filter, Figure 2) also featured diminishing contrast in the raw images which translated either to poor detection of photoblinks or worse localization error. With the sandSTORM experimental protocol used here, effective localization requires collection of light from a mixed population of charge states in a spectral region in which the $\mathrm{NV}^{-}$emission does not dominate over $\mathrm{NV}^{\circ}$. Using the 520/40 BP emission filter, corresponding to a region outside of the $\mathrm{NV}^{\mathrm{o}}$ and $\mathrm{NV}^{-}$emission band no photoblinking was observed.

This spectral dependence of sandSTORM image reconstruction suggests the mechanisms for photoblinking are transient conversions between the $\mathrm{NV}^{\circ}$ and $\mathrm{NV}^{-}$charge states. Owing to the light intensity used in this work, $1.04 \times 10^{6} \mathrm{~W} \mathrm{~cm}^{-2}$, photoinduced electron transfer is thought to be the main driver of charge state conversion and correspondingly photoblinking. Indeed, previous reports [39-41] demonstrate a photoinduced switch from the $\mathrm{NV}^{\circ}$ to the $\mathrm{NV}^{-}$state that was linked to ionization of Nitrogen donors proximal to NVs which involved electron transfer from nearby substitutional $\mathrm{N}$ was to the NV centre. Further support for this hypothesis is work on the photophysics of the NV that has shown the defects' charge state is dynamically modulated between $\mathrm{NV}^{\circ}$ and $\mathrm{NV}^{-}$during illumination with green light by Aslam, Waldherr, Neumann, Jelezko and Wrachtrup [40]. Specifically, their work looked at the dynamics of charge carrier diffusion and trapping that demonstrated efficient production of holes from green laser induced conversion between $\mathrm{NV}^{\circ}$ and $\mathrm{NV}^{-}$. Their findings demonstrate the charge state of NVs is stable in the presence of free electrons and by observing the distribution of trapped charge after local photoionization NVs were found to be effective hole traps, resistant to conduction electrons. They considered the $\mathrm{NV}^{-}$hole capture rate and the $\mathrm{NV}^{\circ}$ electron capture rate in terms of the charge state conversion rate [42]. An additional mechanism causing photoblinking includes $\mathrm{NV}^{-}$ ionization into $\mathrm{NV}^{\circ}$ which is known to depend on the laser power used and the excitation wavelength. Direct ionization of $\mathrm{NV}^{-}$requires photon energies higher than $2.6 \mathrm{eV}$ which is not possible using the $561 \mathrm{~nm}$ illumination in the work here, corresponding to , $2.21 \mathrm{eV}$. However, ionization may occur via a 2 photon absorption process in which one photon induces a transition to the excited state of the defect while the second photon excites the electron to the conduction band of the diamond [43]. Blinking on the microsecond time scale has been ascribed to a photoconversion process of the $\mathrm{NV}$ itself between the $\mathrm{NV}^{-}$and $\mathrm{NV}^{\circ}$ state whilst blinking occurring at the millisecond time scale has been linked with a two photon charge state conversion process. Although it is difficult to elucidate the exact mechanism(s) driving photoblinking in the work herein, photoionization leading to conversion from the NV to the NVcharge states are thought to dominate.

\section{Super-resolution localization of NDs within neuronal cells}

To further demonstrate the use of sandSTORM in biological systems endocytosed NDs within networks of cortical neuronal cells were imaged, Figure 3. Experimentally NDs were introduced into live cultures of cortical neuronal cells following 14 days of culture and incubated for
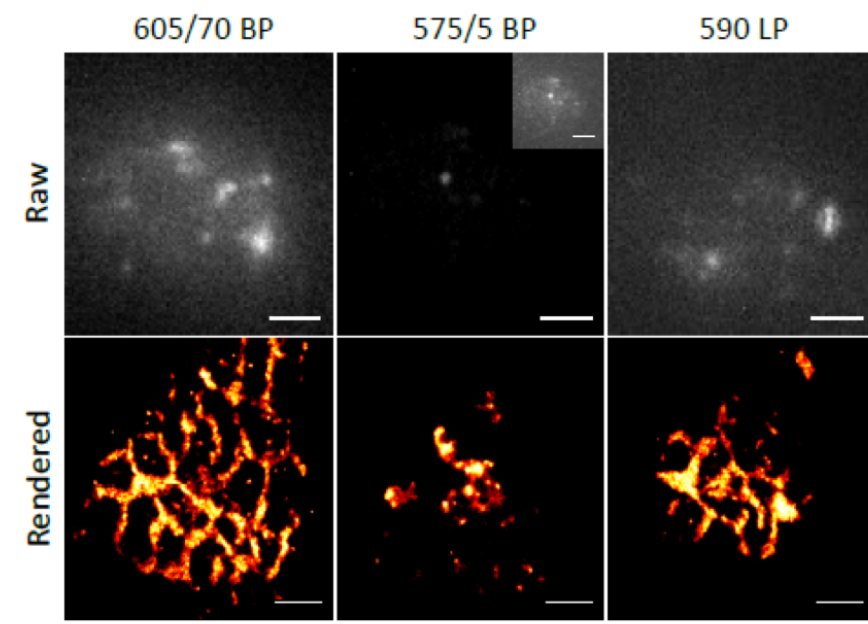

$692 / 40$ BP

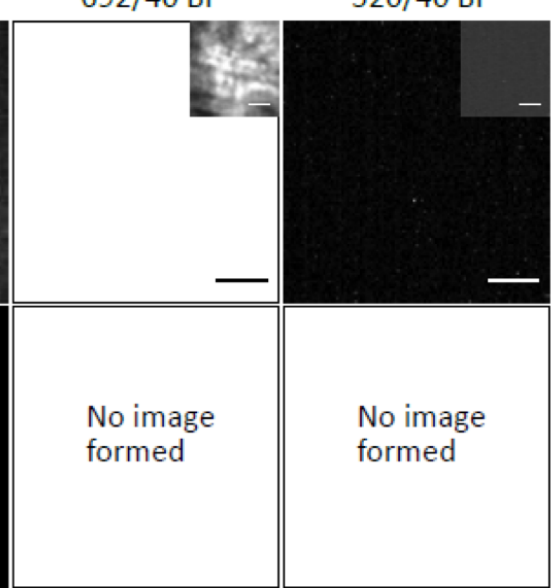

Fig. 2. The spectral band-specific reconstruction of sandSTORM images. Exemplar individual image frames (top row, inserts show images with enhanced contrast) and corresponding sandSTORM reconstructions (bottom row) from experiments carried out using five different emission filters (left to right: $605 \mathrm{~nm} \pm 35 \mathrm{~nm}$ BP, $575 \mathrm{~nm} \pm 2.5 \mathrm{~nm}$ $\mathrm{BP}, 590 \mathrm{~nm}$ LP, $692 \mathrm{~nm} \pm 20 \mathrm{~nm}$ BP, $520 \mathrm{~nm} \pm 20 \mathrm{~nm} \mathrm{BP})$. Scale bar $=2$ $\mu \mathrm{m}$. 


\section{(a)}

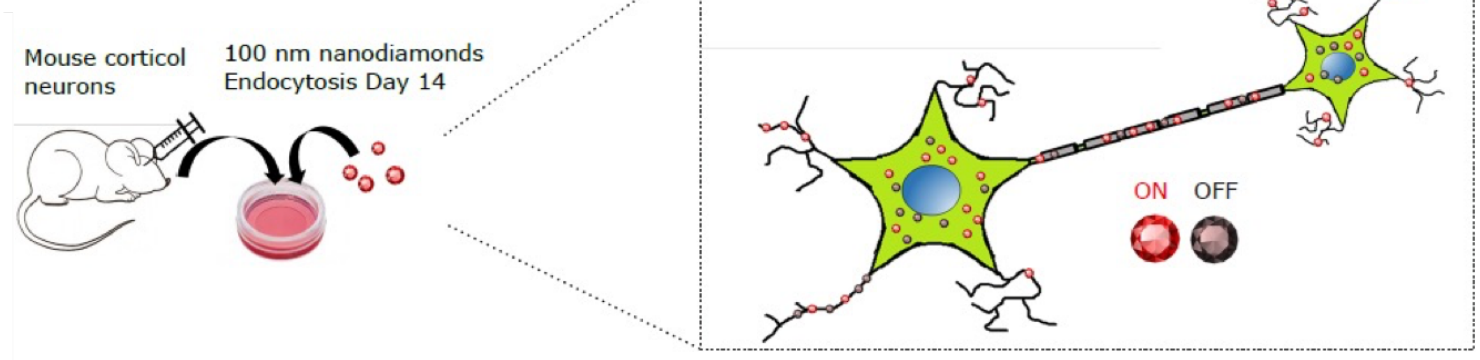

Fig. 3. Imaging internal spaces of cultured neurons with sandSTORM. (a) Schematic of experimental protocol for incorporation of NDs within live neuronal cells. (b) corresponding immunofluorescence, (c) diffraction limited (scale bar $10 \mu \mathrm{m})$ and (d) sandSTORM (scale bar $2 \mu \mathrm{m})(\mathrm{d})$ images of fixed cells (scale bar $2 \mu \mathrm{m}$ ).
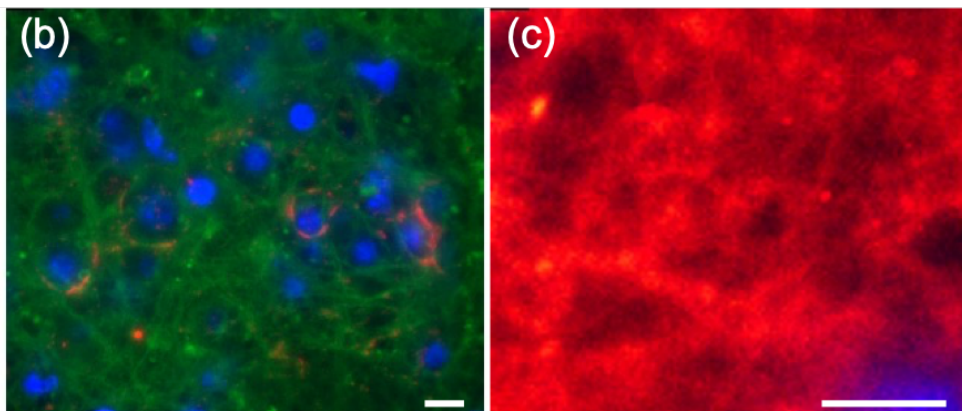

24 hours to allow cell mediated endocytosis of NDs to occur, as depicted in Figure 3a. Cells were subsequently fixed for performing counter-stain immunolabelling to identify cell nuclei and axons and the relative localization of NDs to be visualized (Figure 3b). Fixation was optional as sandSTORM with internalized NDs was compatible with the living culture maintained in PBS. Diffraction limited (Figure 3c) and sandSTORM (Figure 3d) images of NDs were obtained which depict the structural organization of the thread-like structures of axons within the neuronal network, indicating co-localization of NDs with axons. The enhanced localization achieved with sandSTORM as compared to the diffraction limited fluorescent image is apparent and additionally confirms the photoblinking capacity of NVs is retained once internalized in cells. These results further demonstrate that, in addition to targeting the NDs to protein targets via antibodies, they can be directly used to monitor both endocytotic pathways (with potential utility in visualizing targeted drug delivery) and visualize nanoscale intracellular spaces within living cells.

\section{$\underline{\text { NV blinking in different electrolyte solutions }}$}

Factors affecting NV photoblinking were investigated with an emphasis on the chemical environment local to NDs. Herein, spatially localized blinking events were tracked from NDs which have labelled RyR in a muscle tissue section (similar to region shown in Figure 1), immersed sequentially in five different electrolyte aqueous solutions (Hydrochloric acid $(\mathrm{HCl})$, acetic acid $\left(\mathrm{CH}_{3} \mathrm{COOH}\right)$, sodium peroxide $(\mathrm{NaOH})$, sodium chloride $(\mathrm{NaCl})$, phosphate buffered saline (PBS)). Figure 4a displays a subset of time course data for each electrolyte solution studied representative of the number of blinking events recorded per image frame (40,000 frames, $10 \mathrm{~ms}$ exposure time, 5 repeats), extracted using a threshold method (Supplementary Figure 1). The results indicate a dependence of photoblinking on the electrolyte solution in terms of both the number of blinking events per frame and the frequency of events over time (see also Supplementary Movie). To investigate this further, the blinking on and off times for each solution were extracted from the timecourse analysis of the raw image data. Figure $4 \mathrm{~b}$ displays the mean 'on' and 'off' times for each solution as a bar chart. Mean 'off' times were found to be significantly (ANOVA, $\mathrm{p}<0.01$ ) affected by the choice of electrolyte with values ranging between $0.1 \mathrm{~s}$ and $1 \mathrm{~s}$. The variation in

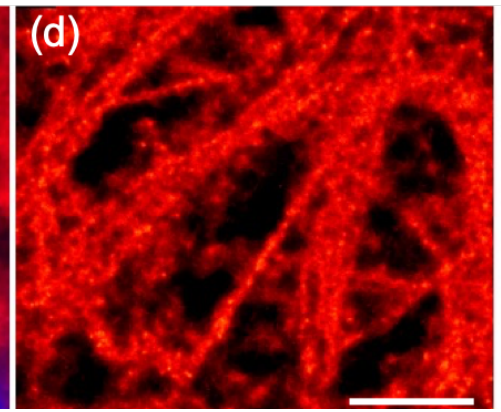

mean 'on' times across electrolytes was comparatively low (26 ms to 32 $\mathrm{ms})$. Notably the mean 'on' and 'off' times differ by up to two orders of magnitude. This finding is in contrast to previous studies of $\mathrm{NV}$ photoblinking which report comparable on and off times however such studies consider single NVs and use higher temporal resolution than the $10 \mathrm{~ms}$ exposure time in the current work. Moreover, the detection of blinking events is contingent of sufficient image contrast between the on and off states which may further complicate detection of events, particularly over the $10 \mathrm{~ms}$ integration time used here.

To investigate the temporal patterns of NV photoblinking and the underlying mechanisms, histograms depicting the probability distribution of 'on' and 'off' times were produced and fitted using a single exponential decay function, see Figure 5 and Supplementary Figure 2. The exponential nature of the probability distribution supports the hypothesis of charge state conversion owing to photoionization and is distinct from previous reports suggesting photoblinking is driven by electron tunneling and thermally dependent processes which characteristically follow a power law dependence. The exponent for each decay curve was also calculated. This revealed variations across the electrolytes used for both 'on' and 'off' times. Indeed, these differences were found to be at least an order of magnitude in the decay exponent between the corresponding 'on' and 'off' times for each electrolyte, readily apparent from the semi-log plots (Figure 5c and 5d). Collectively the experimental results suggest the chemical environment local to NVs strongly affects photoblinking and it is hypothesized that this may arise from a combination of effects including changes in radiative and nonradiative decay paths and lifetimes along with ligand induced shifts in the energies of the dark and bright exciton states [44]. It is further noted that blinking arising from charge state conversion between $\mathrm{NV}^{-}$and $\mathrm{NV}^{\circ}$ via two photon process, initiated by long term photoionization, is liable to be affected by the local chemical environment owing to inhibited recombination in the presence of charge traps near to and within the ND that prevents an efficient diffusion of charge carriers [43]. Moreover, electronic interactions between the NVs and surrounding molecules, including bond-building/breaking and charge transfer, will strongly affect the NV electronic states and correspondingly photoblinking [45]. 
bioRxiv preprint doi: https://doi.org/10.1101/2020.05.20.106716; this version posted May 21, 2020. The copyright holder for this preprint (which was not certified by peer review) is the author/funder, who has granted bioRxiv a license to display the preprint in perpetuity. It is made available under aCC-BY-NC-ND 4.0 International license.

Narayanasamy et al., 18 MAY 2020 - preprint copy - BioRxiv

Experiments presented in Figure 4a also revealed photoblinking could be dynamically adjusted by simply changing the solution in which NDs were imaged. Over the experimental time frame used here photoblinking was not observed from NDs under dry conditions, in freshly deionized water or in media containing significant proportions of glycerol. Further, photoblinking within PBS was found to be independent of oxygen scavengers or reducing agents, typically used in techniques similar to dSTORM. In summary, the data support an environmental-based sensitivity in photoblinking behaviour of NVs which could be further exploited to actively accelerate blinking and correspondingly reduce the duration of sandSTORM measurements.

\section{Comparison between sandSTORM and dSTORM}

The usefulness of sandSTORM as a SMLM technique was evaluated through direct comparison with images of RyRs obtained using dSTORM, with particular emphasis on localization accuracy and image acquisition time. Figure 6 displays rendered dSTORM (Figure 6a) and sandSTORM (Figure 6b) images of RyRs from the same muscle cryosection. Qualitatively, the features seen in both images agree with the expected structural organization of RyR arrays as reported in previous dSTORM studies [37]. To quantitatively compare sandSTORM and dSTORM modalities, histograms were produced to assess the lateral event localization error (Figure 6c) along with Fourier Ring Correlation analysis as a measure of image resolution and as a means to assess the number of image frames and hence imaging time required to attain subdiffraction resolution (Figure 6d). Figure 6c shows comparable lateral localization error for sandSTORM and dSTORM methods (modes of $18 \mathrm{~nm}$ and $\sim 20 \mathrm{~nm}$ respectively) but the range of localization errors for sandSTORM is markedly smaller than in dSTORM (95\% of the events within a range of $32 \mathrm{~nm}$ and $62 \mathrm{~nm}$ ) in similar samples. As a SMLM modality, the faster photoblinking of sandSTORM translates to quicker completion of the image of similar samples. Fourier Ring Correlation (FRC) values which, based on the implementation by Nieuwenhuizen, et al. [46], reports the momentary resolution of the image was used to characterize the temporal course of the image formation. In Figure $6 \mathrm{~d}$, the FRC value reached $95 \%$ of its minimum at $154 \mathrm{~s}$ with sandSTORM at an $\sim 3$-fold speed advantage over the $465 \mathrm{~s}$ for dSTORM. The time course of reconstructing the geometrical features of the image was examined by plotting the Pearson correlation coefficient between the momentary image and the reconstruction from the final (30,000 frames) image (inset). $90 \%$ of the maximum correlation value was within $1000 \mathrm{~s}$ (16 min) for sandSTORM and $\sim 3000 \mathrm{~s}(50 \mathrm{~min})$ for dSTORM. Collectively the results shown in Figures $6 \mathrm{c}$ and $6 \mathrm{~d}$ demonstrate sandSTORM techniques enable attainment of super-resolution images significantly faster than dSTORM opening up the possibility for live cell imaging.

Photobleaching is a known common limitation of dSTORM that compromises localization accuracy over time owing to a reduction in the number of blinking events. Studies carried out herein demonstrate this limitation can be overcome through the use of NVs as fluorescent probes via the sandSTORM method. Experimental results are depicted in Figure 6e which enables a comparison between the number of blinking events recorded over time for sandSTORM and ASTORM. It is apparent that over the time period studied dSTORM suffered from photobleaching, a feature which depends heavily on the redox environment in the imaging field, whilst sandSTORM was resistant to such bleaching. This demonstrates a further strength of sandSTORM and the comparative suitability of sandSTORM for long-term or repeated imaging of samples as compared to dSTORM. As a further assessment of the two STORM methods the number of photons associated with individual blinking events was compared with the number of background photons in raw image frames.

The lower excitation power required for sandSTORM (less than two orders of magnitude compared to dSTORM; see Table 1) offers the added

(a)
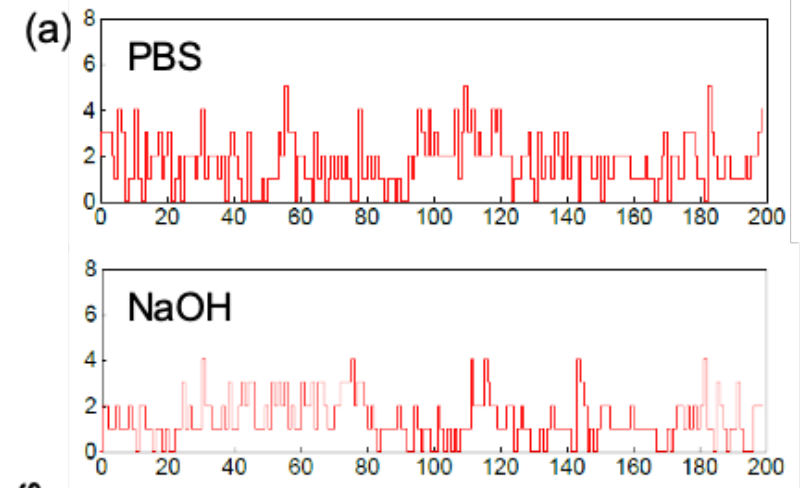

竞
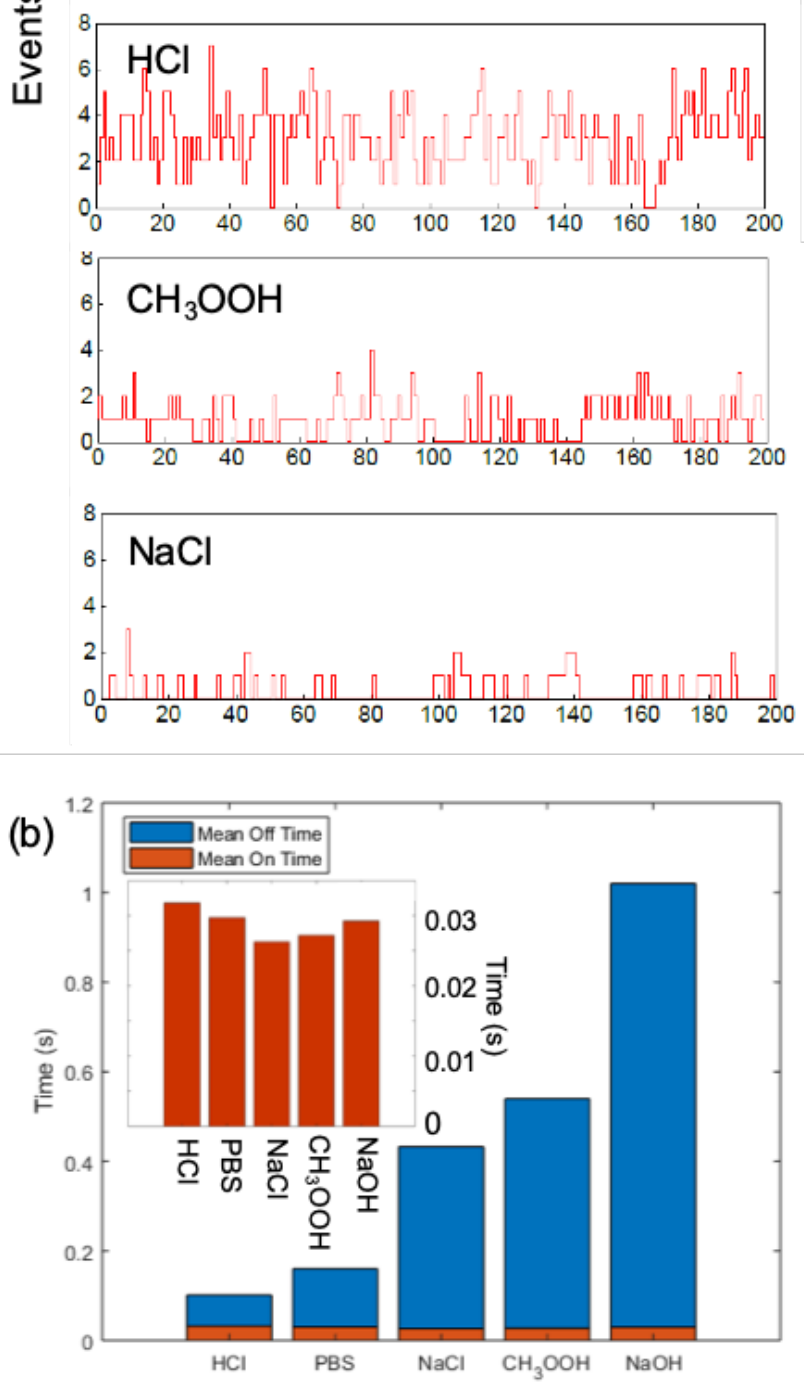

Fig. 4. Effect of electrolyte medium on the temporal patterns of photoblinking in sandSTORM. (a)Time traces of blinking events per frame for 200 frames and (b) mean ON time (expanded detail) and OFF time for a single event in 5 different electrolyte solutions. 
bioRxiv preprint doi: https://doi.org/10.1101/2020.05.20.106716; this version posted May 21, 2020. The copyright holder for this preprint (which was not certified by peer review) is the author/funder, who has granted bioRxiv a license to display the preprint in perpetuity. It is made available under aCC-BY-NC-ND 4.0 International license.

Narayanasamy et al., 18 MAY 2020 - preprint copy - BioRxiv

benefit of a background photon count which is $~ 100$-times lower in sandSTORM compared to that of dSTORM (Figure 6f). This avoids evoking of intrinsic autofluorescence which is a hallmark of large cells and optically-thick tissues and, proportionally reduces the contribution of out-of-focus photoblinking to preserve the contrast of the in-focus blink events.

(a)

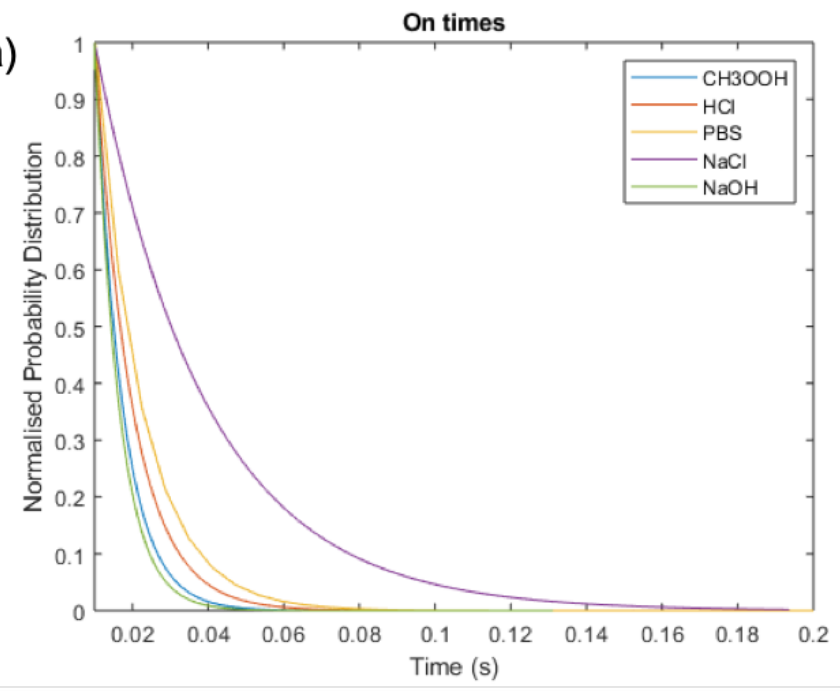

(c)

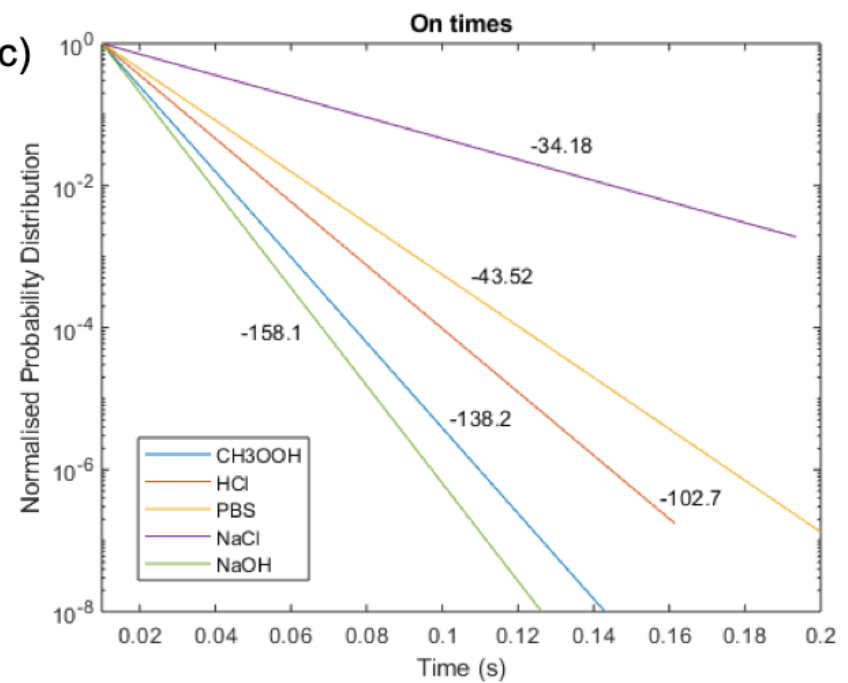

is the correspondence between the location at which the peak in the distribution for sandSTORM events occurs with ND size in Figure $6 \mathrm{~g}$. Differences in the spatial distribution of blinking events between the two methodologies is also seen from inspection of consecutive image frames for sandSTORM (Figure 6h; top row) and dSTORM (bottom row) and in the corresponding kymographs, depicting the fluorescent emission at
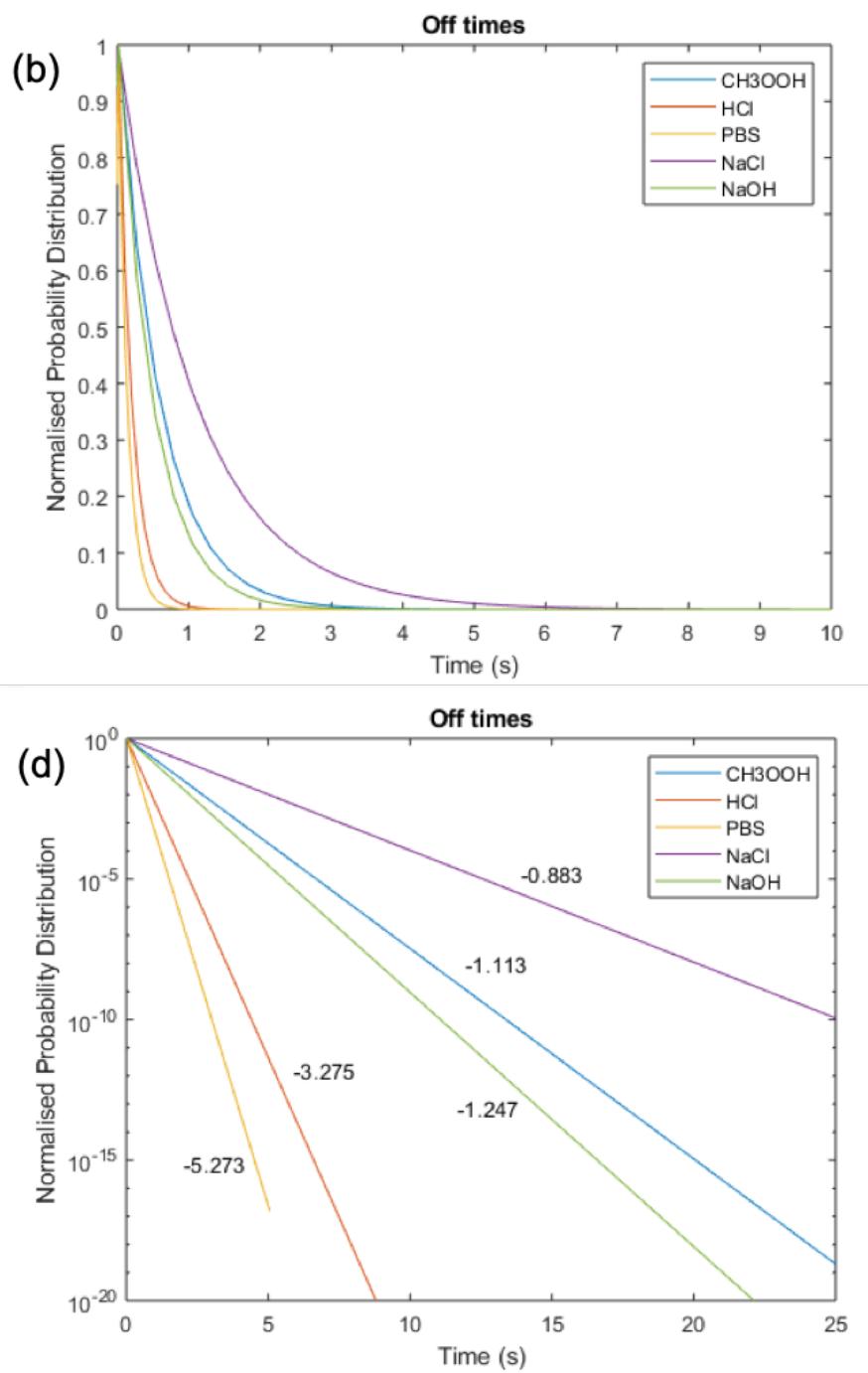

Fig. 5. Temporal parameters of self-activated NV photoblinking in different electrolyte environments. Normalized probability distributions of (a) 'on'times and (b) 'off'-times in $\mathrm{CH}_{3} \mathrm{COOH}, \mathrm{HCl}, \mathrm{PBS}, \mathrm{NaCl}$ and $\mathrm{NaOH}$, shown as fitted mono-exponential decay functions. (c \& d) The respective probability distributions shown as semi-log plots.

The spatial distribution of blinking events was also investigated for both sandSTORM and dSTORM. In typical dSTORM experiments, the spatial distribution of blinking events associated with the aromatic fluorophores used within a given field of illumination, assuming uniformity of illumination, is random. It is hypothesized here that the physical size of individual NDs within a field of illumination will have a bearing on the spatial distribution of blinking events. To investigate this a histogram analysis of the nearest-neighbor distances between events localized within the same image frame was performed for sandSTORM and dSTORM data (Figure 6g) using a multi-emitter localization protocol optimized specifically for STORM data acquired with sCMOS cameras [47]. The histograms demonstrate a broader distribution of nearest neighbors for dSTORM as compared to sandSTORM. Of particular note fixed positions in the image frame over time.

Finally, a comparison of the characteristics of sandSTORM and dSTORM techniques is given in Table 1 . This comparison highlights the advantages of sandSTORM particularly in relation to imaging speed, compatibility with existing biological protocols and suitability for long term imaging within live cells. 
bioRxiv preprint doi: https://doi.org/10.1101/2020.05.20.106716; this version posted May 21, 2020. The copyright holder for this preprint (which was not certified by peer review) is the author/funder, who has granted bioRxiv a license to display the preprint in perpetuity. It is made available under aCC-BY-NC-ND 4.0 International license.

Narayanasamy et al., 18 MAY 2020 - preprint copy - BioRxiv

(a)

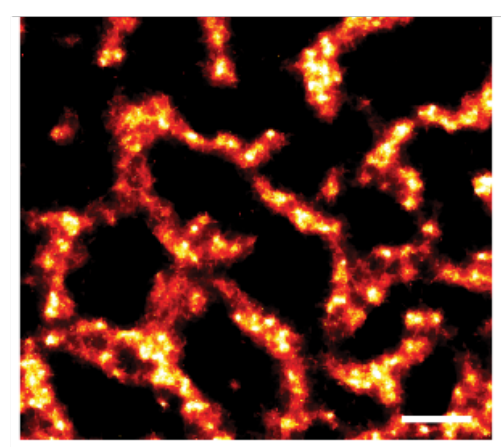

(d)

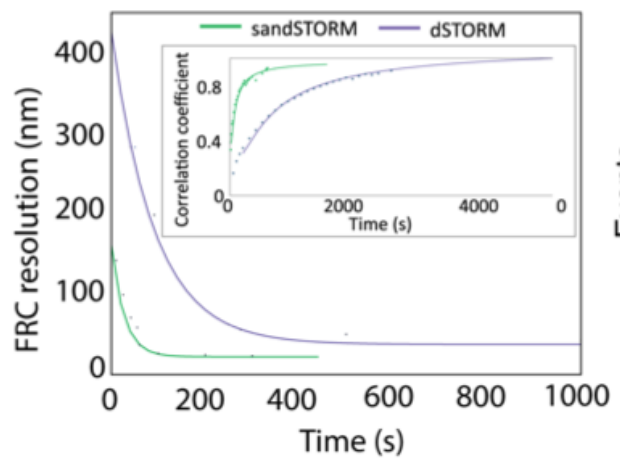

(g)

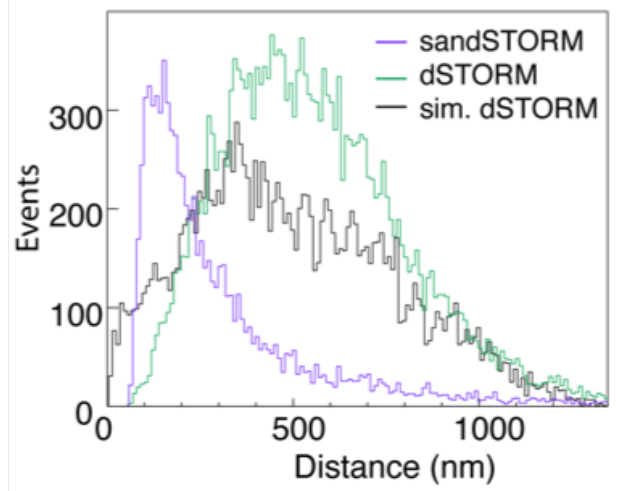

(b)

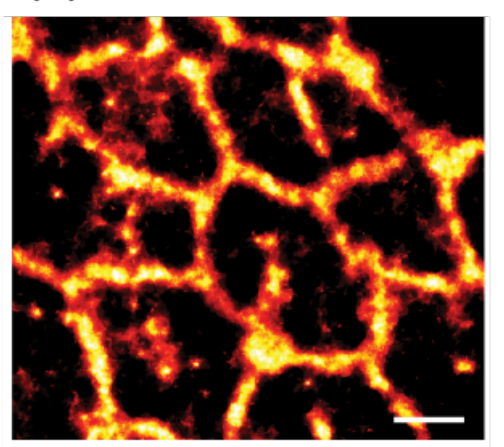

(e)

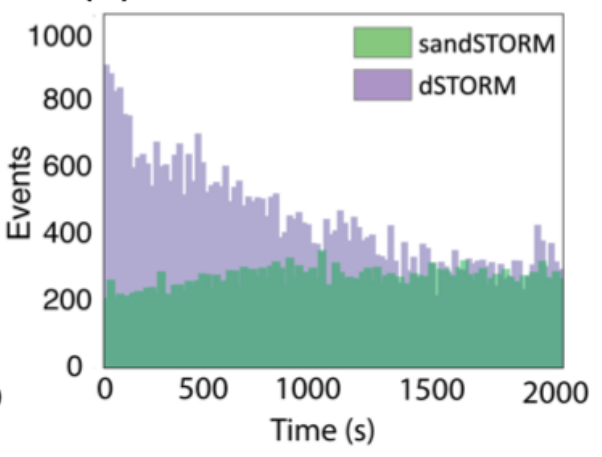

(c)

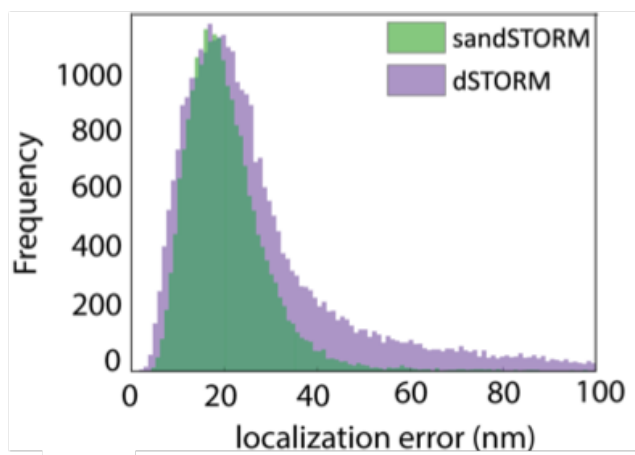

(f)

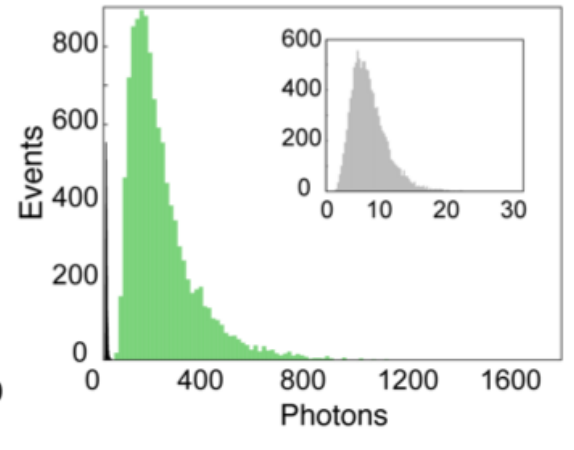

(h) sandSTORM

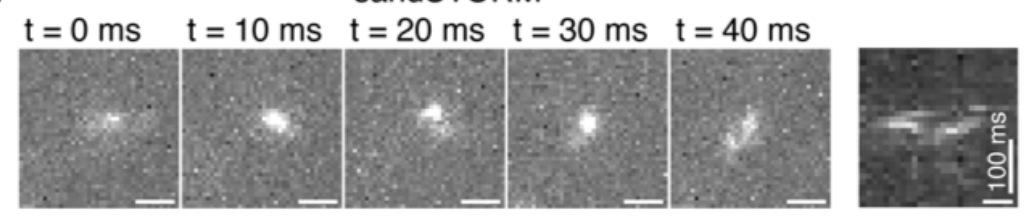

dSTORM

$\mathrm{t}=0 \mathrm{~ms} \quad \mathrm{t}=50 \mathrm{~ms} \quad \mathrm{t}=100 \mathrm{~ms} \mathrm{t}=150 \mathrm{~ms} \mathrm{t}=200 \mathrm{~ms}$

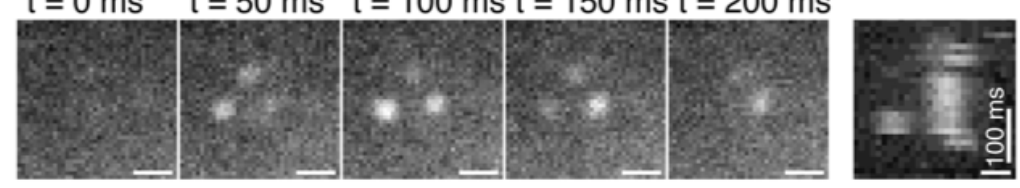

Fig. 6. Comparison between sandSTORM and dSTORM. Rendered (a) dSTORM and (b) sandSTORM images of RyR labelling in fixed skeletal muscle tissue. Overlay plots show (c) histograms of the localization error of events, (d) temporal change in the resolution estimated with Fourier Ring Correlation (inset : time function of Pearson correlation coefficient relative to final image) and (e) temporal histogram of localization event counts for sandSTORM (green) and dSTORM (purple). (f) Overlaid frequency histograms of background photon count of dSTORM (green) and sandSTORM (grey; axes rescaled in inset). (g) Overlaid histograms of nearest neighbor distance for each event localized in a given frame in sandSTORM, dSTORM and events simulated to be spatially-random within the image frame (sim. dSTORM). (h) Serial frames of sandSTORM (upper row) and dSTORM (lower) image sequences illustrating the spatially-linked ND photoblink events. Panel on right shows corresponding $x$-t kymograph of the events through time (in vertical dimension). Scale bars: (a\&b) $1 \mu \mathrm{m}$, (h) $400 \mathrm{~nm}$

\section{Conclusion}

Herein, a method for rapid SMLM that harnesses the self-activated photoblinking of NVs within NDs has been demonstrated. Specifically, the newly introduced method, sandSTORM, has been successfully applied to the study of two distinct and important biological systems, namely membrane receptors within tissue sections and networks of neuronal cells. The observed photoblinking of NV emission was concluded to be driven primarily by photoionization as a result of continuous illumination of samples with light from a $561 \mathrm{~nm}$ laser. Owing to the observed kinetics and spectral dependence of blinking it is considered that conversion from the $\mathrm{NV}^{\circ}$ to the $\mathrm{NV}^{-}$charges state is the dominant effect being observed alongside a two-photon initiated conversion from the $\mathrm{NV}^{-}$to the $\mathrm{NV}^{0}$ charge state. Interestingly it was shown that blinking could be accelerated through changes in the electrolyte in the buffer solution the biological sample was imaged in. This manifests itself as a reduction in blinking off time and is thought to arise as a result of electronic interactions between the NVs and 
Narayanasamy et al., 18 MAY 2020 - preprint copy - BioRxiv

surrounding molecules, including bond-building/breaking and charge transfer.

Major advantages of sandSTORM over existing SMLM methods include the high frame rates super-resolution localization can be achieved at (100 fps (sandSTORM) compared with $0.1 \mathrm{fps}$ (dSTORM)), the simple protocols for incorporation of NDs within samples, the readily accessible experimental implementation within commercial fluorescent microscope and the temporal stability of photoluminescence enabling long term and replicate studies to be performed. Overall, sandSTORM is a SMLM technique with the potential to transform super-resolution imaging within biological systems with particular applications in live cell imaging owing to the fast imaging times, biologically favourable properties of NDs and compatibility of the experimental protocols with existing microscopy platforms in wide use within biological settings.

\begin{tabular}{|c|c|c|}
\hline & sandSTORM & dSTORM \\
\hline Marker durability & $\begin{array}{l}\text { Atomic defects resistant to photobleaching and } \\
\text { biodegradation }\end{array}$ & $\begin{array}{l}\text { Organic fluorophores susceptible to photobleaching and } \\
\text { degradation. }\end{array}$ \\
\hline Imaging speed & $\sim 3-5$ minutes per image; time sampling $\leq 10 \mathrm{~ms}$ & $\sim 20-30$ minutes per image; time sampling $\geqslant 50 \mathrm{~ms}$ \\
\hline Sample medium & $\begin{array}{l}\text { Widely used physiologically compatible aqueous } \\
\text { electrolyte solutions. }\end{array}$ & Buffers require $\mathrm{O}_{2}$ scavengers and/or reducing agents. \\
\hline $\begin{array}{l}\text { Photoactivation/ } \\
\text { excitation }\end{array}$ & $\begin{array}{l}\text { Photoblinking observed at excitation powers of the order } \\
\text { of } 10^{5} \mathrm{~W} \mathrm{~cm}^{-2} \text { to } 10^{6} \mathrm{~W} \mathrm{~cm}^{-2} \text {. }\end{array}$ & $\begin{array}{l}\text { Excitation intensity } \geqslant 10^{7} \mathrm{~W} \mathrm{~cm} \text { to } 10^{9} \mathrm{~W} \mathrm{~cm}^{-2} \\
\text { required, risk of sample phototoxicity. }\end{array}$ \\
\hline Spectra of emission & $\begin{array}{l}\text { Photoblinking observed over spectral band encompassing } \\
\mathrm{NV}^{\circ} \text { and } \mathrm{NV}^{-} \text {emission. }\end{array}$ & $\begin{array}{l}\text { Emission band dependent on spectral properties of } \\
\text { fluorophore. }\end{array}$ \\
\hline
\end{tabular}

Table 1. Comparison of key imaging characteristics of sandSTORM and dSTORM techniques.

\section{Materials and Methods}

\section{Immunohistochemistry}

The extensor digitorum longus (EDL) muscles were dissected from fresh carcasses of healthy male Wistar Crl rats weighing $\sim 200 \mathrm{~g}$ euthanized for heart tissue dissection in Prof Derek Steele's laboratory. Muscles were dissected with intact tendons before pinning out in standard Ringer's saline solution. The muscles were immediately fixed in $2 \%$ paraformaldehyde (PFA; w/v) in phosphate buffered saline (PBS) for 1 hour at $4{ }^{\circ} \mathrm{C}$. Fixed muscles were washed in fresh PBS for 10 minutes and cryoprotected in PBS containing 30\% sucrose. They were frozen in methyl butane cooled in liquid nitrogen, mounted onto a Leica CM 1900 cryostat set to $-25^{\circ} \mathrm{C}$ using OCT compound (Tissue-Tek, USA) and cut into $10 \mu \mathrm{m}$ thick cryosections. Sections were mounted onto number 1.5 glass coverslips (Menzel-Glaser; Germany) coated with $0.05 \%(\mathrm{w} / \mathrm{v})$ poly-L-lysine (Sigma-Aldrich, MO).

Coverslips were attached to the underside of custom-made acrylic stage adapters using silicone elastomer Pinkysil (Barnes, New Zealand) such that an open chamber is formed with the tissue section was positioned in it. Prior to staining, the sections were briefly hydrated and then blocked with Image-iT FX Signal Enhancer (Thermo Fisher, MA) for 1 hour at room temperature $\left(\sim 21^{\circ} \mathrm{C}\right)$. The incubating the primary antibodies in the antibody incubation buffer overnight at $4^{\circ} \mathrm{C}$. Coverslips and sections were rinsed in fresh PBS in three 10-min steps prior to the application of the secondary antibodies for 2 hours at room temperature. Three further rinsing steps were applied prior to immersing the sections in the imaging buffer.

\section{ND conjugation to secondary antibodies}

$100 \mathrm{ul}$ of NDs of 50 or $100 \mathrm{~nm}(1 \mathrm{mg} / \mathrm{mL})$ were dispersed in $400 \mathrm{ul} 1 \mathrm{M}$ HEPES buffer at $\mathrm{pH}$ 5. Ethyl dimethylaminopropyl carbodiimide (EDC; Sigma; $0.04 \mathrm{M}$ ) and N-hydrosuccinimide (NHS; Sigma; $0.01 \mathrm{M}$ ) was added into the ND suspension and sonicated for 1 hour in a sonicator bath. The suspension was centrifuged at $18000 \mathrm{rcf}$ for 10 minutes to pellet NDs and the supernatant was discarded. Centrifugation was repeated with 1x PBS three times to wash NDs. 500 ul of PBS was added and sonicated to disperse ND pellet. $20 \mathrm{ul}$ of secondary antibody (AffiniPure goat anti-mouse IgG or AffiniPure goat anti-rabbit; Jackson Immuno Research, USA) was added and incubated at $4 \mathrm{oC}$ overnight. The suspension was then centrifuged at $18000 \mathrm{rcf}$ for 10 minutes at $4 \mathrm{oC}$ and washed with PBS once. The suspension was dispersed in $400 \mathrm{ul}$ of PBS and $0.05 \%$ sodium azide (Sigma).

All animal studies were conducted in compliance with the ethics and animal welfare in place in the University of Nottingham, in accordance to the 1986 Animals (Scientific Procedures) act. 128

\section{Neuronal cell culture}

C57/BL6 mouse embryos in E16-E17 developmental stage were culled and their brains removed. The brain cortices were dissected, and the meninges separated under a dissection microscope. The tissue was further incubated in Hanks Balanced Salt Solution (HBSS, Ca2+ and Mg2+-free; Gibco) with $1 \mathrm{mg} / \mathrm{ml}$ trypsin and $5 \mathrm{mg} / \mathrm{ml}$ DNAseI (both Sigma) at $37 \mathrm{oC} / 5 \% \mathrm{CO} 2$ for 30 minutes. Following treatment with $0.05 \%(\mathrm{v} / \mathrm{v})$ trypsin inhibitor (Life Technologies), the tissue was washed in Neurobasalmedia (Gibco) and $5 \mathrm{mg} / \mathrm{ml}$ DNAseI was added before mechanical dissociation of the tissue. The cells were finally washed in Neurobasal media and spun down at 250x g for 5 minutes. Dissociated cells were further resuspended in Neurobasal media supplemented with 1x GlutaMax and 2\% (v/v) B-27 (both Gibco) and seeded in $35 \mathrm{~mm} \mu$ Dishes high (Ibidi) with or without diamond plate at $1.25 \times 105 / \mathrm{cm} 2$. Neuron cortical networks were allowed to develop for 14 days before incubation with the NDs. 100nm red fluorescent NDs (fND biotech) were added to cultures at a concentration of $30 \mathrm{ug} / \mathrm{ml}$ to allow cell-mediated endocytosis of the NDs. 
bioRxiv preprint doi: https://doi.org/10.1101/2020.05.20.106716; this version posted May 21, 2020. The copyright holder for this preprint (which was not certified by peer review) is the author/funder, who has granted bioRxiv a license to display the preprint in perpetuity. It is made available under aCC-BY-NC-ND 4.0 International license.

Narayanasamy et al., 18 MAY 2020 - preprint copy - BioRxiv

Cortical neurons containing NDs were fixed 24 hours later in $4 \%(\mathrm{w} / \mathrm{v})$ paraformaldehyde (3.6\% sucrose (w/v), 1x PBS, 5mM MgCl2, pH 7.4; ThermoFisher) for 30 minutes at room temperature, washed with $10 \mathrm{mM}$ Glycine in PBS and further permeabilized in PBS/Glycine-Triton (1x PBS, $10 \mathrm{mM}$ glycine, $0.2 \%$ (v/v) Triton X-100; Sigma) for 20 minutes. The cultures were then blocked for 1 hour at room temperature with 3\% (w/v) BSA in PBS (Sigma), followed by incubation with anti-Gfap $(1: 100$; Abcam) and anti-acetylated tubulin (1:300; clone 611B-1, SigmaAldrich) in blocking buffer at 4oC/overnight. After PBS-Triton 0.1\% (v/v) washes, secondary antibodies Alexa Fluor 488 (1:300; Molecular Probes) were incubated where appropriate for 1 hour.

\section{Materials and solutions}

Red fluorescent NDs of $\sim 50 \mathrm{~nm}$ diameters with a population of NV0 and NV- were used for these experiments (Catalogue number brFND100; FND Biotech, Taiwan). The Ringer solution used for dissecting the fresh muscle tissue was designed previously [48], and contained $112 \mathrm{mM}$ of $\mathrm{NaCl}, 3.3 \mathrm{mM}$ of $\mathrm{KCl}, 2.5 \mathrm{mM}$ of CaCl2, $1 \mathrm{mM}$ of $\mathrm{MgCl} 2$ and $20 \mathrm{mM}$ of HEPES with the $\mathrm{pH}$ adjusted to 7.4 using $\mathrm{NaOH}$. Primary and secondary antibodies were incubated in PBS containing $0.05 \% \mathrm{NaN} 3$, $2 \%$ bovine serum albumin (Sigma), 2\% normal goat serum (Sigma) and $0.05 \%$ Triton X100 (Sigma). The imaging buffer for dSTORM contained $90 \%$ Glycerol (v/v; Sigma) and $15 \mathrm{mM} \beta$-mercaptoethylamine (Sigma) in $1 \mathrm{xPBS}$ at $\mathrm{pH} 8.1$.

A range of aqueous imaging solutions were teste $\mathrm{d}$ for sandstorm. Four ionic solutions were prepared in distilled deionized water which were $\mathrm{HCl}$ and $\mathrm{CH} 3 \mathrm{COOH}$ solutions were prepared at $\mathrm{pH} 3.45, \mathrm{NaOH}$ at $\mathrm{pH}$ 10.55 , and $\mathrm{NaCl}$ at a concentration of $0.35 \mathrm{mM}$. DD water and PBS $1 \mathrm{x}$ (Sigma) were also used.

Primary antibodies used for immunohistochemistry were mouse monoclonal anti-RyR raised against partial RyR1 of chicken pectoral muscle [49]; MA3-925; Thermo Scientific), Alexa Fluor 680- conjugated highly cross-adsorbed $(\mathrm{H}+\mathrm{L})$ goat anti-mouse $\mathrm{IgG}$ and goat anti-rabbit IgG antibodies were used as secondary antibodies in dSTORM labelling experiments.

\section{Image acquisition}

Samples were imaged on a modified Nikon TE2000 inverted microscope with a Nikon 1.49NA oil-immersion TIRF objective which focused excitation light onto the sample in HiLo configuration [50]. This allowed the illumination of $\sim 8 \mu \mathrm{m}$ wide approximately circular area in-plane and $\sim 5 \mu$ m-deep volume. For imaging Alexa 680 markers (dSTORM), the laser beam from a solid-state $642 \mathrm{~nm}$ laser (Cobalt, Sweden) was focused onto the sample at a power of $\sim 2.0 \times 10^{8} \mathrm{~W} \mathrm{~cm}^{-2}$. Emission light was passed through a Q690LP dichroic mirror (Chroma Technology) and an DC/ET720/60m emission filter (Chroma) and recorded onto a Zyla 5.5 USB3.0 scientific-CMOS camera (Andor Ltd, Belfast) at an integration time of $50 \mathrm{~ms} /$ frame. For sandSTORM, A $561 \mathrm{~nm} 200 \mathrm{~mW}$ solid-state laser (CNI lasers, China) was used in combination with an AT565DC dichroic mirror (Chroma). Emission filters used for sandSTORM were the 605/70 nm 49004ET-Cy3/TRITC (Chroma), 692/40 nm BrightLine (Semrock), HQ520/40 nm (Chroma), 575/5 nm (Semrock), and $590 \mathrm{~nm}$ LP (Brightline) Image acquisition for sandSTORM was set at 10 $\mathrm{ms} /$ frame integration. The power of the $561 \mathrm{~nm}$ excitation at the focal plane was measured to be between $5.0 \times 10^{5}$ and $1.0 \times 10^{6} \mathrm{~W} \mathrm{~cm}^{-2}$. To alter the effective excitation intensity, the laser beam was passed through a motorized set of neutral density filters (Thorlabs; Germany).

\section{Image analysis and reconstruction}

Single molecule events in each image frame were detected using a multithreshold detection algorithm. Each detected event was localized by fitting the event with an adaptive two-dimensional Gaussian model using a least-squares fitting procedure adopted in an algorithm described previously [51]. These algorithms were implemented in freely available Python Microscopy Environment (PyME) software (freely available via www.python-microscopy.org) concurrent to image acquisition on a Lenovo ThinkTower workstation with a quad-core CPU, $16 \mathrm{~Gb}$ of memory and a $1 \mathrm{~Tb}$ SSD. Localized coordinates, time stamps and single molecule fitting parameters were saved in HDF format which allowed statistical analysis of parameters such as the localization error and event localization rate.

Greyscale 32-bit TIFF images encoding local densities of localized events were rendered using a protocol based on Delaunay triangulation [52]. Here, each detected event coordinate was jittered randomly in 3D at an amplitude that was proportional to the average distance between this point and its nearest neighbor prior to the calculation of Delaunay triangulation. Averaging between ten independent triangulations produced a greyscale image at a pixel sampling of $5 \mathrm{~nm} /$ pixel with minimal background where intensity was proportional to the local event density.

The number of events per frame were analyzed using TrackMate in ImageJ [53]. Image stacks $(40,000$ frames, $10 \mathrm{~ms} /$ frame, $\mathrm{N}=3)$ were processed in ImageJ, initially using a rolling ball background subtraction based on a 10-pixel kernel, followed by binary thresholding using the 'minimum' filter to allow visualizing of the 'on' blinking events against a zero baseline for the 'off' times. Nine to ten regions of interest were selected within each experimental repeat, corresponding to the location of NDs separated by a distance greater than the point spread function of the objective. A fluorescence time trace for region was plotted in OriginPro 9.0, and the traces were analyzed using the Peak Analyzer toolbox in Origin, to obtain the initial and end frame numbers for each detected peak (SI Fig. 1). ON times were then calculated by tracking the full duration of each event where peaks were detected, whilst OFF times were calculated by subtracting the end frame value of each peak from the start frame value of the next peak in the trace. Mean on and off times were calculated by taking the average for each of the 9 regions across three separate experimental repeats (total 27-30 NDs).

Analysis of time evolution of resolution: For assessing the resolution at a given time point in the image acquisition, the localized coordinates of events were binned into alternating time blocks of 2000 frames up to the time point of interest. The alternating time blocks are combined into two independent sub-samples of event coordinates which were used for calculating the Fourier ring correlation, in PyME as described by [46]. The point at which the spatial frequency of the FRC intersects with $1 / 7$ of the peak correlation was taken as the resolution at that time point.

Completion of the image acquisition, in addition to reaching the finest achievable resolution, requires optimal sampling of the marker population in the field of view. To assess this, the image rendered from the cumulative of the localized events up to each time point was subjected to a retrospective Pearson correlation with a final version of the rendered image, implemented in PyME. The time point at which the Pearson's correlation coefficient exceeds 0.95 of the final image was taken as the 'time to maximum correlation'. 
bioRxiv preprint doi: https://doi.org/10.1101/2020.05.20.106716; this version posted May 21, 2020. The copyright holder for this preprint (which was not certified by peer review) is the author/funder, who has granted bioRxiv a license to display the preprint in perpetuity. It is made available under aCC-BY-NC-ND 4.0 International license.

Narayanasamy et al., 18 MAY 2020 - preprint copy - BioRxiv

\section{Nearest neighbor analysis and simulation of synthetic STORM data}

dSTORM and sandSTORM events were localized within a $8 \times 8 \mu \mathrm{m}$ window using a custom-written multi-emitter localization algorithm described previously [54] and available in PyME. Two-dimensional coordinates and the time stamps (in image frame number) of the events localized with this routine were analyzed further in a custom-written script. Where each image frame recorded multiple localized events, the distance from the centroid of each event to that of its nearest neighboring concurrent event was recorded and plotted as frequency histograms.

To simulate a random blinking spatiotemporal pattern, a synthetic image series was rendered via PyME (as described previously in the supplementary section of [7]) using the rendered sandSTORM images as a starting map of spatial density of markers. The localization and image acquisition parameters (e.g. localization error, sigma of each fit, amplitude and series length) were set to mimic dSTORM experimental data. The simulation parameters included pre-set values such as average event number per pixel of the starting image (1.0), mean event intensity (1000 photons), mean event background intensity (200 photons), mean event duration (150 ms), mean number of detections per marker (2.0) and number of image frames $(30,000)$.

\section{Statistical analysis}

Statistical analysis was conducted using OriginPro 9.0. A one-way ANOVA statistical analysis was performed for the ON and OFF times for events $(\mathrm{N}=3)$. P values were denoted as $* \mathrm{p}<0.05$ to indicate statistical significance between groups.

\section{End Matter}

\section{Supporting information}

The authors wish to dedicate this publication to Dr Simon Levett, our beloved colleague and friend, who sadly recently passed away. Simon was a fundamental member of our research team who was a source of constant support and scientific insight.

Supporting Information is available from the figure supplement or from the author.

\section{Author Contributions and Notes}

Experiments were conceived by K.K.N., I.J., J.C.P. \& M.L.M. Experiments were performed, and data were acquired by K.K.N., J.C.P \& R.M-R.. Data were analyzed by K.K.N., I.J., J.C.P. \& MLM, interpreted by K.K.N., I.J., J.C.P. \& M.LM. The manuscript was written by K.K.N., I.J, M.L.M \& J.C.P.

The authors declare no conflict of interest.

\section{Acknowledgments}

The authors thank the Wellcome Trust (Seed Award awarded to IJ; Ref 207684/Z/17/Z awarded to IJ) and the European Research Council (ERC) (ERC Consolidator Award, TransPhorm, grant number 23432094 awarded to MLM) for the funding which supported this research. Also acknowledged, are Tim Munsey for his assistance with dissecting and sectioning the muscle tissue used for imaging experiments, Dr David Baddeley (University of Auckland) and Prof Christian Soeller (University of Exeter) for helpful discussions on the adaptation of PyME for the image analysis presented in this manuscript and Drs David
Simpson and Liam Hall (University of Melbourne) for useful discussion on NV charge state dynamics.

\section{References}

1. Schroeder, L. K.; Barentine, A. E. S.; Merta, H.; Schweighofer, S.; Zhang, Y.; Baddeley, D.; Bewersdorf, J.; Bahmanyar, S., J Cell Biol 2019, 218 (1), 83-96. DOI 10.1083/jcb.201809107.

2. Shim, S. H.; Xia, C.; Zhong, G.; Babcock, H. P.; Vaughan, J. C.; Huang, B.; Wang, X.; Xu, C.; Bi, G. Q.; Zhuang, X., Proc Natl Acad Sci U S A 2012, 109 (35), 13978-83. DOI 10.1073/pnas.1201882109.

3. Leterrier, C.; Potier, J.; Caillol, G.; Debarnot, C.; Rueda Boroni, F.; Dargent, B., Cell Rep 2015, 13 (12), 2781-93. DOI 10.1016/j.celrep.2015.11.051.

4. Mikhaylova, M.; Cloin, B. M.; Finan, K.; van den Berg, R.; Teeuw, J.; Kijanka, M. M.; Sokolowski, M.; Katrukha, E. A.; Maidorn, M.; Opazo, F.; Moutel, S.; Vantard, M.; Perez, F.; van Bergen en Henegouwen, P. M.; Hoogenraad, C. C.; Ewers, H.; Kapitein, L. C., Nat Commun 2015, 6, 7933. DOI 10.1038/ncomms8933.

5. Beliveau, B. J.; Boettiger, A. N.; Avendano, M. S.; Jungmann, R.; McCole, R. B.; Joyce, E. F.; Kim-Kiselak, C.; Bantignies, F.; Fonseka, C. Y.; Erceg, J.; Hannan, M. A.; Hoang, H. G.; Colognori, D.; Lee, J. T.; Shih, W. M.; Yin, P.; Zhuang, X.; Wu, C. T., Nat Commun 2015, 6, 7147. DOI 10.1038/ncomms8147.

6. Xu, J.; Ma, H.; Jin, J.; Uttam, S.; Fu, R.; Huang, Y.; Liu, Y., Cell Rep 2018, 24 (4), 873-882. DOI 10.1016/j.celrep.2018.06.085.

7. Jayasinghe, I.; Clowsley, A. H.; Lin, R.; Lutz, T.; Harrison, C.; Green, E.; Baddeley, D.; Di Michele, L.; Soeller, C., Cell Rep 2018, 22 (2), 557-567. DOI 10.1016/j.celrep.2017.12.045.

8. Pageon, S. V.; Tabarin, T.; Yamamoto, Y.; Ma, Y.; Nicovich, P. R.; Bridgeman, J. S.; Cohnen, A.; Benzing, C.; Gao, Y.; Crowther, M. D.; Tungatt, K.; Dolton, G.; Sewell, A. K.; Price, D. A.; Acuto, O.; Parton, R. G.; Gooding, J. J.; Rossy, J.; Rossjohn, J.; Gaus, K., Proc Natl Acad Sci U S A 2016, 113 (37), E5454-63. DOI 10.1073/pnas.1607436113.

9. Sahl, S. J.; Hell, S. W.; Jakobs, S., Nat Rev Mol Cell Biol 2017, 18 (11), 685-701. DOI 10.1038/nrm.2017.71.

10. Rust, M. J.; Bates, M.; Zhuang, X., Nat Methods 2006, 3 (10), 793-5. DOI 10.1038/nmeth929.

11. Baddeley, D.; Jayasinghe, I. D.; Cremer, C.; Cannell, M. B.; Soeller, C., Biophys J 2009, 96 (2), L22-4. DOI 10.1016/j.bpj.2008.11.002.

12. Heilemann, M.; van de Linde, S.; Schuttpelz, M.; Kasper, R.; Seefeldt, B.; Mukherjee, A.; Tinnefeld, P.; Sauer, M., Angew Chem Int Ed Engl 2008, 47 (33), 6172-6. DOI 10.1002/anie.200802376.

13. Sage, D.; Pham, T. A.; Babcock, H.; Lukes, T.; Pengo, T.; Chao, J.; Velmurugan, R.; Herbert, A.; Agrawal, A.; Colabrese, S.; Wheeler, A.; Archetti, A.; Rieger, B.; Ober, R.; Hagen, G. M.; Sibarita, J. B.; Ries, J.; Henriques, R.; Unser, M.; Holden, S., Nat Methods 2019, 16 (5), 387-395. DOI 10.1038/s41592-019-0364-4.

14. Samanta, S.; Gong, W.; Li, W.; Sharma, A.; Shim, I.; Zhang, W.; Das, P.; Pan, W.; Liu, L.; Yang, Z.; Qu, J.; Kim, J. S., Coordination Chemistry Reviews 2019, 380, 17-34.

15. Folling, J.; Bossi, M.; Bock, H.; Medda, R.; Wurm, C. A.; Hein, B.; Jakobs, S.; Eggeling, C.; Hell, S. W., Nat Methods 2008, 5 (11), 9435. DOI 10.1038/nmeth.1257.

16. Dempsey, G. T.; Vaughan, J. C.; Chen, K. H.; Bates, M.; Zhuang, X., Nat Methods 2011, 8 (12), 1027-36. DOI 10.1038/nmeth.1768.

17. van de Linde, S.; Loschberger, A.; Klein, T.; Heidbreder, M.; Wolter, S.; Heilemann, M.; Sauer, M., Nat Protoc 2011, 6 (7), 991-1009. DOI 10.1038/nprot.2011.336. 
bioRxiv preprint doi: https://doi.org/10.1101/2020.05.20.106716; this version posted May 21, 2020. The copyright holder for this preprint (which was not certified by peer review) is the author/funder, who has granted bioRxiv a license to display the preprint in perpetuity. It is made available under aCC-BY-NC-ND 4.0 International license.

Narayanasamy et al., 18 MAY 2020 - preprint copy - BioRxiv

18. Karlsson, J. K. G.; Laude, A.; Hall, M. J.; Harriman, A., Chemistry 2019, 25 (65), 14983-14998. DOI 10.1002/chem.201904117.

19. Jungmann, R.; Avendano, M. S.; Woehrstein, J. B.; Dai, M.; Shih, W. M.; Yin, P., Nat Methods 2014, 11 (3), 313-8. DOI 10.1038/nmeth.2835.

20. Oi, C.; Gidden, Z.; Holyoake, L.; Kantelberg, O.; Mochrie, S.; Horrocks, M.; Regan, L., LIVE-PAINT: Super-Resolution Microscopy Inside Live Cells Using Reversible Peptide-Protein Interactions. bioRxiv: 2020.

21. Jung, S. R.; Fujimoto, B. S.; Chiu, D. T., Curr Opin Chem Biol 2017, 39, 64-73. DOI 10.1016/j.cbpa.2017.06.004.

22. Peeters, Y.; Vandenberg, W.; Duwe, S.; Bouwens, A.; Lukes, T.; Ruckebusch, C.; Lasser, T.; Dedecker, P., Sci Rep 2017, 7 (1), 10470. DOI 10.1038/s41598-017-09666-4.

23. Bon, P.; Bourg, N.; Lecart, S.; Monneret, S.; Fort, E.; Wenger, J.; Leveque-Fort, S., Nat Commun 2015, 6, 7764. DOI 10.1038/ncomms8764.

24. Peng, X.; Huang, B.; Pu, R.; Liu, H.; Zhang, T.; Widengren, J.; Zhan, Q.; Agren, H., Nanoscale 2019, 11 (4), 1563-1569. DOI 10.1039/c8nr08986h.

25. He, H.; Liu, X.; Li, S.; Wang, X.; Wang, Q.; Li, J.; Wang, J.; Ren, H.; Ge, B.; Wang, S.; Zhang, X.; Huang, F., Anal Chem 2017, 89 (21), 11831-11838. DOI 10.1021/acs.analchem.7b03567.

26. Wang, Y.; Fruhwirth, G.; Cai, E.; Ng, T.; Selvin, P. R., Nano Lett 2013, 13 (11), 5233-41. DOI 10.1021/nl4026665.

27. Culley, S.; Tosheva, K. L.; Matos Pereira, P.; Henriques, R., Int J Biochem Cell Biol 2018, 101, 74-79. DOI 10.1016/j.biocel.2018.05.014.

28. Cox, S.; Rosten, E.; Monypenny, J.; Jovanovic-Talisman, T.; Burnette, D. T.; Lippincott-Schwartz, J.; Jones, G. E.; Heintzmann, R., Nat Methods 2011, 9 (2), 195-200. DOI 10.1038/nmeth.1812.

29. Ouyang, W.; Aristov, A.; Lelek, M.; Hao, X.; Zimmer, C., Nat Biotechnol 2018, 36 (5), 460-468. DOI 10.1038/nbt.4106.

30. Pfender, M.; Aslam, N.; Waldherr, G.; Neumann, P.; Wrachtrup, J., Proc Natl Acad Sci U S A 2014, 111 (41), 14669-74. DOI 10.1073/pnas.1404907111.

31. Su, L. J.; Fang, C. Y.; Chang, Y. T.; Chen, K. M.; Yu, Y. C.; Hsu, J. H.; Chang, H. C., Nanotechnology 2013, 24 (31), 315702. DOI 10.1088/0957-4484/24/31/315702.

32. Arroyo-Camejo, S.; Adam, M. P.; Besbes, M.; Hugonin, J. P.; Jacques, V.; Greffet, J. J.; Roch, J. F.; Hell, S. W.; Treussart, F., ACS Nano 2013, 7 (12), 10912-9. DOI 10.1021/nn404421b.

33. Han, K. Y.; Kim, S. K.; Eggeling, C.; Hell, S. W., Nano Letters 2010, 10 (8), 3199-3203. DOI 10.1021/nl102156m.

34. Barbiero, M.; Castelletto, S.; Gan, X.; Gu, M., Light Sci Appl 2017, 6 (11), e17085. DOI 10.1038/lsa.2017.85.

35. Gu, M.; Cao, Y.; Castelletto, S.; Kouskousis, B.; Li, X., Opt Express 2013, 21 (15), 17639-46. DOI 10.1364/OE.21.017639.
36. Watanabe, T. M.; Fukui, S.; Jin, T.; Fujii, F.; Yanagida, T., Biophysical Journal 2010, 99 (7), L50-L52. DOI 10.1016/j.bpj.2010.07.036.

37. Jayasinghe, I. D.; Munro, M.; Baddeley, D.; Launikonis, B. S.; Soeller, C., J R Soc Interface 2014, 11 (99). DOI 10.1098/rsif.2014.0570.

38. Doherty, M. W.; Manson, N. B.; Delaney, P.; Jelezko, F.; Wrachtrup, J.; Hollenberg, L. C. L., Physics Reports 2013, 528 (1), 1-45.

39. Manson, N. B.; Harrison, J. P., Diamond and Related Materials 2005, 14 (10), 1705-1710.

40. Aslam, N.; Waldherr, G.; Neumann, P.; Jelezko, F.; Wrachtrup, J., New Journal of Physics 2013, 15 (1), 013064. DOI 10.1088/13672630/15/1/013064.

41. Gaebel, T.; Domhan, M.; Wittmann, C.; Popa, I.; Jelezko, F.; Rabeau, J.; Greentree, A.; Prawer, S.; Trajkov, E.; Hemmer, P. R.; Wrachtrup, J., Applied Physics B 2006, 82 (2), 243-246. DOI 10.1007/s00340-0052056-2.

42. Dhomkar, S.; Zangara, P. R.; Henshaw, J.; Meriles, C. A., Phys Rev Lett 2018, 120 (11), 117401. DOI 10.1103/PhysRevLett.120.117401.

43. Achard, J.; Jacques, V.; Tallaire, A., Journal of Physics D: Applied Physics 2020.

44. Fomenko, V.; Nesbitt, D. J., Nano Lett 2008, 8 (1), 287-93. DOI $10.1021 /$ nl0726609.

45. Petit, T.; Pfluger, M.; Tolksdorf, D.; Xiao, J.; Aziz, E. F., Nanoscale 2015, 7 (7), 2987-91. DOI 10.1039/c4nr06639a.

46. Nieuwenhuizen, R. P.; Lidke, K. A.; Bates, M.; Puig, D. L.; Grunwald, D.; Stallinga, S.; Rieger, B., Nat Methods 2013, 10 (6), 557-62. DOI 10.1038/nmeth.2448.

47. Lin, R.; Clowsley, A. H.; Jayasinghe, I. D.; Baddeley, D.; Soeller, C., Opt Express 2017, 25 (10), 11701-11716. DOI 10.1364/OE.25.011701.

48. Jayasinghe, I. D.; Launikonis, B. S., J Cell Sci 2013, 126 (Pt 17), 404858. DOI 10.1242/jcs.131565.

49. Airey, J. A.; Beck, C. F.; Murakami, K.; Tanksley, S. J.; Deerinck, T. J.; Ellisman, M. H.; Sutko, J. L., J Biol Chem 1990, 265 (24), 14187-94.

50. Tokunaga, M.; Imamoto, N.; Sakata-Sogawa, K., Nat Methods 2008, 5 (2), 159-61. DOI 10.1038/nmeth1171.

51. Baddeley, D.; Jayasinghe, I. D.; Lam, L.; Rossberger, S.; Cannell, M. B.; Soeller, C., Proc Natl Acad Sci U S A 2009, 106 (52), 22275-80. DOI 10.1073/pnas.0908971106.

52. Baddeley, D.; Cannell, M. B.; Soeller, C., Microsc Microanal 2010, 16 (1), 64-72. DOI 10.1017/S143192760999122X.

53. Tinevez, J. Y.; Perry, N.; Schindelin, J.; Hoopes, G. M.; Reynolds, G. D.; Laplantine, E.; Bednarek, S. Y.; Shorte, S. L.; Eliceiri, K. W., Methods 2017, 115, 80-90. DOI 10.1016/j.ymeth.2016.09.016.

54. Baddeley, D., Biophysical Journal 2014, 106 (2), 605a. DOI 10.1016/j.bpj.2013.11.3347. 